\title{
Class number formula for certain imaginary quadratic fields
}

\author{
N. L. Wang ${ }^{1, ~}$, T. Arai ${ }^{1,2}$ \\ ${ }^{1}$ Dept. of Appl. Math., Shangluo Univ. Shangluo, 726000, PRC \\ ${ }^{2} \mathrm{Grad}$. School of Advances Tech. Kinki Univ., Iizuka, 820-8555, Japan
}

Email address:

wangnianliangshangluo@aliyun.com (N. L. Wang)

\section{To cite this article:}

N. L. Wang, T. Arai. Class Number Formula for Certain Imaginary Quadratic Fields. Pure and Applied Mathematics Journal. Special Issue: Abridging over Troubled Water---Scientific Foundation of Engineering Subjects. Vol. 4, No. 2-1, 2015, pp. 1-6.

doi: 10.11648/j.pamj.s.2015040201.11

\begin{abstract}
In this note we shall show how Carlitz in 1954 could have reached an analogue of the Voronoi congruence in the more difficult case of $p \equiv 1(\bmod 4): h(-4 p) \equiv \mathrm{B}_{(p+1) / 2}\left(x_{4}\right)(\bmod p)$, where $\mathrm{B}_{(p+1) / 2}\left(x_{4}\right)$ is the generalized Bernoulli number with $x_{4}$ being the Kronecker symbol associated to the Gaussian field $\mathrm{Q}(\sqrt{-}-4)$.
\end{abstract}

Keywords: Class Number Formula, Short Interval Character Sum, Generalized Bernoulli Number, Euler Number

\section{Introduction}

Throughout this paper let $p$ be an odd prime and let $\alpha \in \mathrm{N}$. Let $\varphi(n)$ denote Euler's function counting the number of integers between 1 and $n$ relatively prime to $n$. Then $\varphi\left(p^{\alpha}\right)=p^{\alpha}-p^{\alpha-1}$ and $\varphi(p)=p-1$.

In the case $p \equiv 1(\bmod 4)$ the Legendre symbol $\chi_{p}(a)=(a \mid p)$ is the real primitive even Dirichlet character to the modulus $p$ associated to the real quadratic field $\mathrm{Q}(\sqrt{\mathrm{p}})$ and the Kronecker symbol $\chi_{-4 p}(a)=\chi_{4} \chi_{p}(a)=\chi_{4}(a)(a \mid p)$ is the real primitive odd character associated to $\mathrm{Q} \sqrt{-4 \mathrm{p}})$, while in the case $p \equiv 1(\bmod 4)$, the Legendre symbol is the real primitive odd character associated to the imaginary quadratic field $\mathrm{Q}(\sqrt{-\mathrm{p}})$ and the Kronecker symbol $\chi_{4} \chi_{p}(a)=\chi_{4}(a)(a \mid p)$ is the real primitive even character associated to the real quadratic field $\mathrm{Q}(\sqrt{4 \mathrm{p}})$, where $\chi_{4}$ indicates the real primitive Dirichlet character mod 4 associated to the Gaussian field $\mathrm{Q}(i)$.

For a Dirichlet character $\chi \bmod q$, let $\tau(\chi)=\sum_{a \bmod q} \chi(a) e^{2 \text { pia/q }}$ be the normalized Gauss sum.

Let $L(s, \chi)$ denote the Dirichlet $L$-function associated to $\chi$. For a non-principal $\chi$, the series for $L(s, \chi)$ is convergent for $\sigma=\operatorname{Re} s>0$ and we may speak of the value
$L(1, \chi)$. Throughout in what follows, whenever we refer to a character, we mean an odd character (hence necessarily non-principal). Let $h(d)$ denote the class number of the imaginary quadratic field $\mathrm{Q} \sqrt{\mathrm{d}})$ with discriminant $d<0$, and let $\chi_{d}(\cdot)=(d \mid \cdot)$ be the corresponding Kronecker character. In this sense $\chi_{4}$ above is $\chi_{-4}$ but we use the notation $\chi_{4}$. Then Dirichlet's class number formula reads

$$
h(d)=w \sqrt{|d|} L\left(1, \chi_{d}\right) /(2 \pi)
$$

where $w$ is the number of roots of 1 in $\mathrm{Q}(\sqrt{\mathrm{d}})$.

For the imaginary quadratic field $\mathrm{Q}(\sqrt{-4 \mathrm{p}}), \quad p \equiv 1(\bmod 4)$, Eq. (1) reads

$$
h(-4 p)=2 \sqrt{4 p} L\left(1, \chi_{-4 p}\right) /(2 \pi)=2 B_{1}\left(\chi_{-4 p}\right)
$$

where $B_{n}(\chi)$ is the $n$th generalized Bernoulli number to be introduced in Section 4.

We recall that Kummer, who trying to prove the Fermat last theorem, found an equivalent condition to the regularity of a prime to be the non-divisibility of the numerators of Bernoulli numbers $B_{2}, B_{4}, \cdots, B_{p-3}$ by $p([1$, Theorem $2, \mathrm{p}$. 417]). Since $E_{n}=-2 B_{n+1}\left(\chi_{4}\right) /(n+1)$, the simplest generalized Bernoulli number, the non-divisibility of $E_{(p-1) / 4}$ by $p$ is to be interpreted to correspond to some regularity of a certain class number type stuff, and it is so as 
shown in Theorem 1.

Also recall the following fact comparable to Eq. (2)

Proposition 1 [Voronoi] For $p \equiv 3(\bmod 4)$, we have

$$
h(-p) \equiv-2 B_{(p+1) / 2}(\bmod p)
$$

where $B_{(p+1) / 2}$ is the $(p+1) / 2$-th Bernoulli number.

The congruence Eq. (3) follows from Voronoi's work and was taken up as exercises in Borevic and Safarevic [1], and more recently recasted by Ireland and Rosen [2].

If in the case $p \equiv 3(\bmod 4)$, we consider two quadratic fields $\mathrm{Q} \sqrt{4 \mathrm{p}})$ and $\mathrm{Q} \sqrt{-\mathrm{p}})$ as sub-fields of $\mathrm{Q}(i, \sqrt{4 \mathrm{p}})$, and interpret Eq. (3) to mean the congruence of the class number to an easily calculable number, then it is quite natural to ask for the counterpart of the congruence Eq. (3) for the imaginary quadratic field $\mathrm{Q} \sqrt{-4 \mathrm{p}})$ in the case $p \equiv 1(\bmod 4)$, since it would be the one in the case of two subfields of $\mathrm{Q}(\sqrt{-1}, \sqrt{4 \mathrm{p}})$. The answer is given by the following

Theorem 1. For $p \equiv 1(\bmod 4)$, the counterpart of Voronoi's congruence Eq. (3) reads

$$
h(-4 p) \equiv-2 B_{(p+1) / 2}\left(\chi_{4}\right)(\bmod p)
$$

This follows immediately from the Carlitz-would-have-proved result Eq. (9) in the special case of $\alpha=1$ :

Theorem 2. Carlitz in 1954 would have proved for $p \equiv 1(\bmod 4)$

$$
2 h(-4 p)=4 \sum_{s=1}^{(p-1) / 4}(s \mid p) \equiv E_{\varphi\left(p^{\alpha}\right) / 2}\left(\bmod p^{\alpha}\right)
$$

in particular

$$
2 h(-4 p)=4 \mathrm{~S}_{1 / 4,0} \equiv E_{(p-1) / 2}(\bmod p)
$$

where $\mathrm{S}_{1 / 4,0}$ is the 1/4-th character sum.

There have appeared several papers claiming that the Euler number $E_{(p-1) / 2}$ in the case $p \equiv 1(\bmod 4)$ is the least positive residue $\bmod p$, and it a fortiori that it is not divisible by $p$, answering the question posed by Guy [3,B45] as unsolved. Indeed, it was settled in 1972 already. This unfortunate situation has been somehow streamlined by Liu [4] but not fully. It is only in [5] that the proper formulation of the results were made $([5,(2.26)])$ which still lacks the proper interpretation in our Theorem 1 above. Also, although [5] is the most advanced and contains many other expressions involving the values $L(2 r+1, \chi)$, the most interesting case is the case $\alpha=1$ and the higher power case gives just complicated expressions. E. g. for $\alpha=2,1=2$ $[5,(2.25)]$ reads

$$
p^{2} h(-4 p)+B_{3}\left(\chi_{-4 p}\right) / 3 \equiv 4 B_{(p+5) / 2} /(p+5)(\bmod p)
$$

In view of this, we confine ourselves to the congruence for the $\varphi\left(p^{\alpha}\right) / 2$-th Euler number $E_{\varphi\left(p^{\alpha}\right) / 2}$ considered representatively in [8], on pp. 283-291, it is stated

$$
\begin{aligned}
& E_{\phi\left(p^{\alpha}\right) / 2} \equiv 4 \sum_{s=1}^{(p-1) / 4}(s \mid p) \\
& =-2 i \tau\left(\chi_{4} \chi_{p}\right) L\left(1, \chi_{4} \chi_{p}\right) \quad(\bmod p)
\end{aligned}
$$

which is to read Eq.(5).

It follows that the mid-term in Eq.(8) is less than $p$ and the far-left term is a positive integer (in Eq.(8), the far-right term cannot be seen to be a positive integer without appealing to the class number formula), i.e. that $E_{\varphi\left(p^{\alpha}\right) / 2}$ is congruent to the least positive residue $\bmod p$, and a fortiori $E_{\varphi\left(p^{\alpha}\right) / 2}$ is congruent to the least positive residue mod $p$.

Thus deducing a congruence of an Euler number through the class number (or one of its equivalent forms) is in the reverse direction, i. e. rather similar to the related problem of Chowla which asks for an elementary proof of the non-vanisngness of $S_{1 / 1,0}$. Only in the reverse direction of assuming the non-vanishingness, it is proved that the cotangent values are linearly independent over Q(cf. [9]).

In Section 2, we shall show how Carlitz in 1954 could have proved all these results on Euler number congruences, confirming that results on Euler numbers could be obtained as special cases of generalized Bernoulli numbers. We note that this interpretation (in the case $p \equiv 1(\bmod 4))$ is possible only when one considers not only the field $Q(\sqrt{-4 p})$ but also the field $Q(\sqrt{p})$ as subfields of the bicyclic biquadratic field $Q(i, \sqrt{p})$ (cf. [10]). We shall also publicize in Section 3 the important universal method of Yamamoto for expressing the short interval character sums in terms of $\mathrm{L}(1, \chi)$, hence of class numbers, streamlining the situation.

\section{The Carlitz 1954 Proof}

First we state two auxiliary lemmas which are useful in elementary evaluations, the first being the reduction principle, the second the parity principle.

Lemma 1. For any periodic function $\chi$ of period $q$ and any $N \in \mathrm{N}$, we have

$$
\sum_{a=1}^{N} \chi(a)=\left[\frac{N}{q}\right] \sum_{a=1}^{q} \chi(a)+\sum_{a=1}^{N-q[N / q]} \chi(a)
$$

where $N-q[N / q]$ is the least non-negative residue of $N(\bmod q)$.

In particular, if $\chi$ is a non-principal character $\bmod p$, and $N=\beta\left(p^{\alpha}-1\right) \in \mathrm{N}$, then

$$
\sum_{a=1}^{\beta\left(p^{\alpha}-1\right)} \chi(a)=\sum_{a=1}^{\beta(p-1)} \chi(a)
$$


Proof. Eq.(9) follows from the division of the interval:

$$
\sum_{a=1}^{N} \chi(a)=\sum_{b=0}^{\left[\frac{N}{q}\right]} \sum_{c=1}^{q} \chi(c+b q)+\sum_{c=1+q[N / q]}^{N} \chi(c)
$$

$$
\sum_{a=1}^{N}(-1)^{a} f(a)=2 \sum_{\mathrm{a}=1}^{\left[\frac{N}{2}\right]} f(2 a)-\sum_{a=1}^{N} f(a)
$$

and in particular for any Dirichlet character $\chi$ and any integer $j \geq 0$, we have

and Eq.(10) follows from the from the orthogonality of

characters and $\beta\left(p^{\alpha}-1\right)-p\left[\frac{\beta\left(p^{\alpha}-1\right)}{p}\right]=\beta(p-1)$.

Lemma 2.For any function $f$, we have

$$
\sum_{a=1}^{N}(-1)^{a}(2 a)^{j} \chi(2 a)=2^{j}\left(\chi(4) 2^{j+1} S_{1 / 2, j}(\chi)-\chi(2) S_{1 / 1, j}(\chi)\right)
$$

Proof. follows immediately on classifying the values of $a \bmod 2$.

Corollary 1. [9, Theorem 10]

$$
(1-2 \chi(2)) \sum_{a=1}^{N} a \chi(a)=\chi(2) N \sum_{\mathrm{a}=1}^{\left[\frac{N}{2}\right]} \chi(a)
$$

i. e.

$$
(1-2 \chi(2)) S_{1 / 1,1}=\chi(2) N S_{1 / 2,0}
$$

Proof. We note that Eq. (12) reads for a non-principal

Dirichlet character $\bmod N, N$ being odd,

$$
\sum_{a=1}^{N}(-1)^{a} a^{j} \chi(a)=\chi(2) 2^{j+1} S_{1 / 2, j}(\chi)-S_{1 / 1, j}(\chi)
$$

which is the equality in the proof of Funakura. The rest of the proof uses the finite Fourier transform and we refer to [9], completing the proof.

Remark 1. The Dirichlet class number formula Eq. (1) for the imaginary quadratic field in finite form reads (cf. [11, p.53])

$$
h(d)=-\frac{1}{|d|} S_{1 / 1,1}\left(\chi_{d}\right)
$$

in view of

$$
E_{\varphi\left(p^{\alpha}\right) / 2} \equiv 2(-1)^{\frac{p^{\alpha}-1}{2}} \sum_{s=0}^{\left(p^{\alpha}-1\right) / 2}(-1)^{s}(2 s)^{l}(2 s \mid p)^{\frac{m}{\varphi\left(p^{\alpha}\right) / 2}}\left(\bmod p^{\alpha}\right)
$$

$$
E_{\varphi\left(p^{\alpha}\right) / 2} \equiv 2(-1) \sum_{s=0}^{\frac{p^{\alpha}-1}{2}} \sum_{\left(p^{\alpha}-1\right) / 2}(-1)^{s}(2 s)^{l}(2 s \mid p)\left(\bmod p^{\alpha}\right)
$$

Proof. Equation $[15,(1.10)]$ reads for any $r \in \mathrm{N}$,

$$
E_{2 n} \equiv 2(-1)^{r} \sum_{s=0}^{r}(-1)^{s}(2 s)^{2 n}\left(\bmod (2 r+1)^{2}\right)
$$

Choosing $r=\left(p^{\alpha}-1\right) / 2$ and replacing the powers $(2 s)^{m}$ by the Legendre symbol in Eq. (22) leads to Eq. (20).
Remark 2. Note that Eq. (20) is [5,(1.6)] which in turn is a generalization of Zhang and $\mathrm{Xu}[5,1.4, \mathrm{p} .290]$ which is an essential formula in their whole argument.

The case $m$ being an even multiple of $\varphi\left(p^{\alpha}\right) / 2$ is also treated in [5].

Equation [22] appears in Carlitz [15, p.39] who uses a telescoping of the difference equation satisfied by the Euler polynomial. We note that since the exponent of $p$ in (22) is $2 \alpha$, there is a possibility of obtaining the congruence modulo a higher power of $p$ (details will appear elsewhere). 
Proof of Theorem 2. Although in [1] as well as in [2], an appeal to the Euler criterion is made in order to replace the power of an integer by the Legendre symbol, it was used as early as in Carlitz [15, p. 39] who replaces the power $a^{\varphi\left(p^{\alpha}\right) / 2}$ by $(a \mid p) \bmod p^{\alpha}$ in the case $m$ is an odd multiple of $\varphi\left(p^{\alpha}\right) / 2$ in [15, (1.6)]. If he did it in [15, (1.10)] (Lemma 3 above), then he would have immediately obtained Eq.(20). Then by the reduction lemma and the parity lemma, everything boils down to the 1/4-th sum $S_{1 / 4,0}\left(\chi_{p}\right)$ for which he should have known the Dirichlet class number formula Eq. (18) and the proof would have been complete.

\section{Short Interval Character Sums}

In this section we shall just supplement to [16] which contains many useful identities for short interval character sums in terms of the values of the L-function, by restricting those involving class numbers. We have a handy complete table of short interval sums of Kronecker symbols [6] which will suffice for the purpose of this note, but we state the results in slightly more general form. The following is a slight generalization of Yamamoto's theorem.

Lemma 4. ([16, (5.3)]) Let $\beta=t / u, t, u \in \mathrm{N}$, and $0<t \leq u, k \in \mathrm{N} \cup\{0\}$ and let

$$
S_{\beta, k}=S_{\beta, k}(\chi)=\frac{1}{q^{k}} \sum_{0 \leq a \leq \beta q}^{\prime} a^{k} \chi(a)
$$

for a primitive Dirichlet character $\chi \bmod q$, where the prime on the summation sign means that for extremal values $0, \beta q$ of $a$, the corresponding summand is to be halved. Then

$$
\begin{gathered}
S_{\beta, k}=k ! \tau(\chi) \sum_{r=1}^{k} \frac{\beta^{k-r+1}}{(2 \pi i)^{r}(k-r+1) !} \sum_{n=1}^{\infty} \frac{b_{r}(n) \bar{\chi}(n)}{n^{r}}+ \\
\frac{k ! \tau(\chi)}{(2 \pi i)^{k+1}} \sum_{n=1}^{\infty} \frac{b_{k+1}(n) \bar{\chi}(n)}{n^{k+1}}
\end{gathered}
$$

where

$$
\begin{aligned}
& b_{r}(n)=(-1)^{r+1} \chi(-1) \eta^{n t}-\eta^{-n t} \quad(1 \leq r \leq k), \\
& b_{k+1}(n)=(-1)^{k+1} \chi(-1)\left(1-\eta^{n t}\right)+1-\eta^{-n t}
\end{aligned}
$$

and $\eta=e^{2 \pi i / u}$.

Lemma 4 allows us to establish all the short interval results used in section 3 in a unified and stereotyped way.

Lemma 5. For $p \equiv 1(\bmod 4)$, let $\chi_{p}(a)=(a \mid p)$ be the real primitive even character corresponding to $\mathrm{Q}(\sqrt{p})$. Then we have

$$
\begin{aligned}
S_{1 / 4}\left(\chi_{p}\right) & =S_{1 / 4,0}\left(\chi_{p}\right)=\frac{\tau\left(\chi_{p}\right)}{\pi} L\left(1, \chi_{4} \chi_{p}\right) \\
& =-\frac{2 i}{\pi} \tau\left(\chi_{4} \chi_{p}\right) L\left(1, \chi_{4} \chi_{p}\right)
\end{aligned}
$$

and

$$
S_{1 / 2}\left(\chi_{p}\right):=S_{1 / 2,0}\left(\chi_{p}\right)=0 .
$$

On the other hand, for $p \equiv 3(\bmod 4)$, let $\chi_{-p}(a)=(a \mid p)$ be the real primitive odd character corresponding to $\mathrm{Q}_{(\sqrt{-p})}$. Then we have

$$
\begin{aligned}
& \mathrm{S}_{1 / 4}\left(\chi_{-\mathrm{p}}\right)=\mathrm{S}_{1 / 4,0}\left(\chi_{-\mathrm{p}}\right)=\frac{\tau\left(\chi_{-\mathrm{p}}\right)}{2 \pi \mathrm{i}}\left(1+\chi_{-\mathrm{p}}(2)\right) \mathrm{L}\left(1, \chi_{-\mathrm{p}}\right) \\
& \mathrm{S}_{1 / 2}\left(\chi_{-\mathrm{p}}\right):=\mathrm{S}_{1 / 2,0}\left(\chi_{-\mathrm{p}}\right)=\left(2-\chi_{-\mathrm{p}}(2)\right) \frac{\tau\left(\chi_{-\mathrm{p}}\right)}{\pi \mathrm{i}} \mathrm{L}\left(1, \chi_{-\mathrm{p}}\right)
\end{aligned}
$$

Proof. For $p \equiv 1(\bmod 4)$, we apply Lemma 4 with

$$
k=0, \alpha=\frac{1}{4}=\frac{t}{\mathrm{u}}, t=1, u=4, \eta=e^{\pi / 2} .
$$

Then $b_{1}(n)=2 i \chi_{4}(n)$ and

$$
S_{1 / 4}\left(\chi_{p}\right)=\frac{\tau\left(\chi_{p}\right)}{2 \pi i} \sum_{n=1}^{\infty} \frac{2 i \chi_{4}(n) \chi_{p}(n)}{n}
$$

which gives the first equality in Eq.(25). The second follows on substituting $\tau\left(\chi_{4}\right)=i \sqrt{|-4|}=2 i$.

In the same way, we choose

$$
k=0, \alpha=\frac{1}{2}=\frac{t}{u}, t=1, u=2, \eta=e^{2 \pi / 2}=-1,
$$

and Eq. (26) follows.

Now suppose $p \equiv 3(\bmod 4)$. Then $\chi_{-p}(-1)=-1$. Choosing the parameters as

$$
k=0, \alpha=\frac{1}{4}=\frac{t}{u}, t=1, u=4, \eta=e^{\pi / 2}=i,
$$

we deduce Eq. (27) after some calculations.

For $k=0, \alpha=\frac{1}{2}=\frac{t}{u}, t=1, u=2, \eta=e^{\pi i / 2}=-1, \quad$ we obtain

$$
\begin{gathered}
S_{1 / 2}\left(\chi_{-p}\right)=\frac{4 \tau\left(\chi_{-p}\right)}{2 \pi i} \sum_{n=1(\bmod 2)} \frac{\chi_{-p}(n)}{n}= \\
\frac{4 \tau\left(\chi_{-p}\right)}{2 \pi i} \sum_{n=1}^{\infty} \frac{\chi_{-p}(n)[1]_{2}}{n},
\end{gathered}
$$

where $[1]_{2}$ is the principal character mod 2 , whence we may deduce Eq.(28), completing the proof.

Remark 3. Eq.(25) and Eq.(26) already appear in [16, p.282] and Eq. (25) is [8, Lemma 3]. Zhang and Xu [8, Lemma 2] who appealed to the identity valid for a primitive odd character $\chi$ to the conductor $f$ :

$$
S_{1 / 1,1}(\chi)=\frac{1}{f} \sum_{0 \leq a \leq f} a \chi(a)=-\frac{\tau(\chi)}{\pi i} L(1, \bar{\chi})
$$


to prove Eq.(25). However Eq.(29) is a form of Dirichlet's class number formula and is an immediate consequence of Lemma 4: $\alpha=1=\frac{t}{\mathrm{u}}, t=u=1, \eta=1, \quad$ and so that $b_{1}(n)=-2, b_{2}(n)=0$. Hence,

$$
S_{1 / 1,1}(\chi)=-2 \frac{\tau(\chi)}{2 \pi i} \sum_{n=1}^{\infty} \frac{\bar{\chi}(n)}{n}
$$

which gives Eq.(29).

\section{Generalized Bernoulli Numbers}

Let $\chi$ be a primitive character to the modulus $\mathrm{f}$. The $\mathrm{j}$-th generalized Bernoulli number $B_{j}(\chi)$ is defined by

$$
B_{j}(\chi)=f^{j-1} \sum_{a=1}^{f} \bar{\chi}(a) B_{j}\left(\frac{a}{f}\right),
$$

in particular,

$$
E_{n}=-\frac{2}{n+1} B_{n+1}\left(\chi_{4}\right)
$$

(cf. e. g. [17]). On [18, p. 174], Eq. (31) is misstated.

It is known that for $\chi$ odd,

$$
B_{2 j+1}(\chi)=\frac{2(-1)^{j} \tau(\chi)(2 j+1) !}{f(2 \pi / f)^{2 j+1}} L(2 j+1, \chi),
$$

and in particular

$$
B_{1}(\chi)=\frac{\tau(\bar{\chi})}{\pi} L(1, \chi)=\frac{1}{\mathrm{f}} S_{1 / 1,1} .
$$

Carlitz proved

Lemma 6. If $\chi$ is a primitive character to the modulus $f$ and $p$ is a prime such that $p \nmid f$, and $\varphi\left(p^{\alpha}\right) \mid n$, then

$$
\frac{B_{\mathrm{n}+1}(\chi)}{n+1} \equiv \frac{1}{f}(1-\chi(p)) S_{1 / 1,1}\left(\bmod p^{\alpha}\right)
$$

and in particular,

$$
\frac{B_{\mathrm{n}+1}\left(\chi_{4}\right)}{n+1} \equiv 0\left(\bmod p^{\alpha}\right)
$$

In view of Eq. (31), Eq. (35) in the special case $\chi=\chi_{4}$ asserts for $\varphi\left(p^{\alpha}\right) \mid n$ that $E_{n}$ is congruence to 0 or 2 according as $p \equiv 1(\bmod 4)$ or $p \equiv-1(\bmod 4)$, which is an refinement of Nielsen's results, which we state as

Proposition 2. If $\varphi\left(p^{\alpha}\right) \mid 2 n$, then

$$
E_{2 n} \equiv 0 \quad \text { or } 2 \quad\left(\bmod p^{\alpha}\right)
$$

according as $p \equiv 1(\bmod 4)$ or $p \equiv 3(\bmod 4)$;
In particular,

$$
E_{p-1} \equiv 0 \quad \text { or } 2 \quad(\bmod p)
$$

according as $p \equiv 1(\bmod 4)$ or $p \equiv 3(\bmod 4)$.

The proof follows from Eq.(20) with $\varphi\left(p^{\alpha}\right) \mid 2 n$

$\frac{2 n}{\varphi\left(p^{\alpha}\right) / 2}$ being even, in which case we have $[1]_{p}(s)$ in place of the Legendre symbol, $[1]_{p}$ being the principal character $\bmod p$ :

$$
E_{2 n}=2(-1)^{\frac{p^{\alpha}-1}{2}} \sum_{s=1}^{\left[\frac{p^{\alpha}-1}{2}\right]}(-1)^{s}[1]_{p}(s) \quad\left(\bmod p^{\alpha}\right)
$$
[5].

The details of a more complicated case has been treated in

Remark 4. Eq.(36) is due to Carlitz [15], who uses a slightly different methods and also states that the special case Eq.(37) was proved already in 1923 by Nielsen [19, p.273]. The implication of Lemma 6 also would suggest to consider an analogue of the Voronoi congruence Eq.(3) .

\section{Concluding Remarks}

1. As has been completely demonstrated in this note, any short interval character sum may be expressed as a linear combination of $L(1, \chi)^{\prime} \mathrm{s}$ and inevitably in terms of the class number Eq.(1) (as Berndt says, "we give here some proofs of Eq.(5) ([12, p. 261]) that do not involve class number consideration, although, admittedly, the use of $L$-function gives an undeniable link with class numbers.") It therefore follows that any attempt at obtaining a closed form of such a short interval sum in an elementary way bears no credit. Berndt [12, pp.413-445] contains a number of useful formulas, but of course is not exhaustive (cf. formulas in Lemma 5). But now that we have Yamamoto's colossal theory, we are supposed to use it.

2. We notice that in Lemma 5 we consider short interval character sums with polynomial weight, and it a fortiori, of Bernoulli polynomial weight, and the final formulas contain Bernoulli numbers and class numbers of imaginary quadratic fields (cf. Lemma 5; for $p \equiv 1(\bmod 4), \chi_{4} \chi_{p}$ is odd, and for $p \equiv 3(\bmod 4), \chi_{4} \chi_{p}$ is odd $)$ as in Berndt [12, pp. 413-445] and [6]. But Yamamoto also treats the case of Clausen function weight, or what is the same thing, log sin weight. Therefore, it is very intriguing to pursue research on class numbers of real quadratic fields as in Chowla [14].

3 . Considering congruences to the higher prime power modulus is important from $p$-adic theoretic point of view. The results like Eq.(5) or Eq.(36) are not mere generalization of Eq.(10) or Eq.(12), but should be interpreted p-adically. As the example of Shiratani and Yokoyama [20], some relations on Bernoulli or Euler numbers can be deduced by $p$-adic argument (cf. [21] for general treatment). We hope to return to this direction of research elsewhere. 
4. One could use the well-known relation $2 n T_{n}=2 n E_{2 n-2}^{(2)}=2^{2 n}\left(2^{2 n}-1\right) B_{2 n}$ (cf. [19, p. 56]) on the tangent coefficient or the second order Euler numbers to deduce Voronoi congruence. One could try to generalize Voronoi's argument to treat the generalized Bernoulli number.

\section{Acknowledgements}

The authors would like to express their hearty thanks to Professor S. Kanemitsu for enlighting discussions and his patient supervision.

The first author is supported by the Shaanxi Province N.S.F.(2012JM1018) and (2014JM1019) and Shaanxi Edu. Dept. N.S.F.(2013JK0570).

\section{References}

[1] Z. N. Borevic and I. S. Shafarevic, The theory of numbers, 2nd edition, Nauka, Moscow 1972 (in Russian); first edition published in 1964.

[2] K. Ireland and M. Rosen, A Classical Introduction to Modern Number Theory, Springer-Verlag, New York etc., 1990.

[3] R. K. Guy, Unsolved Probelms in Number Theory, 3rd ed. Springer-Verlag, New York etc., 2004.

[4] G. -D. Liu, Generating functions and generalized Euler numbers, doctoral thesis, Kinki University, 2006.

[5] N. -L. Wang, J. -Z. Li and D. -S. Liu, Euler numbers congruences and Dirichlet L-functions, J. Number Theory 129 (2009), 1522-1531.

[6] A. Schinzel, J. Urbanowicz and P. Van Wamelen, Class numbers and short sums of Kronecker symbols, J. Number Theory 78 (1999), 62-84.

[7] J. Szmidt, J. Urbanowicz and D. Zagier, Congruences among generalized Bernoulli numbers, Acta Arith. 71 (1995), 275-278.

[8] W. -P. Zhang and Z.-F. Xu, On a conjecture of the Euler numbers, J. Number Theory 127 (2007), 283-291.
[9] T, Funakura, On Kronecker's limit formula for Dirichlet series with periodic coefficients, Acta Arith. 55 (1990), 59-73.

[10] K. Chakraborty, S. Kanemitsu and T. Kuzumaki, Arithmetical class number formula for certain quadratic fields, Hardy-Ramanujan J. 36 (2013), 1-7

[11] H. Davenport, Multiplicative Number Theory, Markham 1967, second edition Springer-Verlag, New York etc., 1982.

[12] B. C. Berndt, Classical theorems on quadratic residues, Enseign. Math. (2) 22(1976), 261-304.

[13] L. E. Dickson, History of Number Theory, Chelsea, New York 1952.

[14] P. Chowla, On the class number of real quadratic field, J. Reine Angew. Math. 230 (1968), 51-60.

[15] L. Carlitz, A note on Euler numbers and polynomials, Nagoya Math. J. 7 (1954), 35-43.

[16] Y. Yamamoto, Dirichlet series with periodic coefficients, Proc. Intern. Sympos. "Algebraic Number Theory", Kyoto 1976, JSPS, Tokyo 1977, 275-289.

[17] B. C. Berndt, Periodic Bernoulli numbers, summation formulas and applications, Theory and Applications of Special Functions, Richard Askey ed. Academic Press, New York, 1975, 143-189.

[18] L. Carlitz, Arithmetical properties of generalized Bernoulli numbers, J. Reine Angew. Math. 202 (1959), 174-182.

[19] N. Nielsen, Traite elementaire des Nombres de Bernoulli, Gauther-Villars, Paris, 1923, 35-43.

[20] B. C. Berndt, Character analogues of the Poisson and Euler-Maclaurin summation formulas with applications, J. Number Theory 7(1975), 413-445.

[21] K. Shiratani and S. Yokoyama, An application of $p$-adic convolutions, Mem. Fac. Sci., Kyushu Univ. Ser. Math. 36 (1982), 73-83.

[22] L. C. Washington, Introduction to cyclotomic fields, 2nd ed., Springer, New York/Berlin, 1997. 